\title{
Cosmic magnetism with the Square Kilometre Array and its pathfinders
}

\author{
Bryan M. Gaensler \\ Sydney Institute for Astronomy (SIfA), School of Physics, \\ The University of Sydney, NSW 2006, Australia \\ email: bgaensler@usyd.edu.au
}

\begin{abstract}
One of the five key science projects for the Square Kilometre Array (SKA) is "The Origin and Evolution of Cosmic Magnetism", in which radio polarimetry will be used to reveal what cosmic magnets look like and what role they have played in the evolving Universe. Many of the SKA prototypes now being built are also targeting magnetic fields and polarimetry as key science areas. Here I review the prospects for innovative new polarimetry and Faraday rotation experiments with forthcoming facilities such as ASKAP, LOFAR, the ATA, the EVLA, and ultimately the SKA. Sensitive wide-field polarisation surveys with these telescopes will provide a dramatic new view of magnetic fields in the Milky Way, in nearby galaxies and clusters, and in the high-redshift Universe.
\end{abstract}

Keywords. Galaxies: magnetic fields - instrumentation: interferometers - polarimeters - intergalactic medium - ISM: magnetic fields - magnetic fields - radio continuum: general - techniques: polarimetric

\section{Introduction}

The Square Kilometre Array (SKA) $\dagger$ is a concept for a next-generation radio telescope, with a total approximate collecting area of $1 \mathrm{~km}^{2}$ (Schilizzi et al. 2008); an artist's impression of the central core of the SKA is shown in Figure 1. From the outset, the SKA project has been a global effort, and is currently governed by an international consortium of 18 member countries. Two potential sites are being considered for the SKA: near Boolardy station in Western Australia, and in the Karoo region of South Africa.

The SKA will be a highly flexible instrument, covering a broad range of frequencies $(0.07-25 \mathrm{GHz})$, with many different operating modes. At an observing frequency of $1.4 \mathrm{GHz}$, the SKA will have a maximum angular resolution of 0. . 02 and a field of view of 20 square degrees. This latter specification provides incredible survey capability, far exceeding that of any other sensitive radio telescope. A unique capability of radio arrays is that they can begin taking science data science long before the full facility is complete. Operations for the SKA are thus projected to begin in around 2016, with an approximate construction cost of $€ 1.5$ billion.

Five key science projects have been designated for the SKA, as discussed by Gaensler (2004) and Carilli \& Rawlings (2004). Below I summarise recent progress and developments on one of these five key projects, "The Origin and Evolution of Cosmic Magnetism" (see Gaensler et al. 2004). 


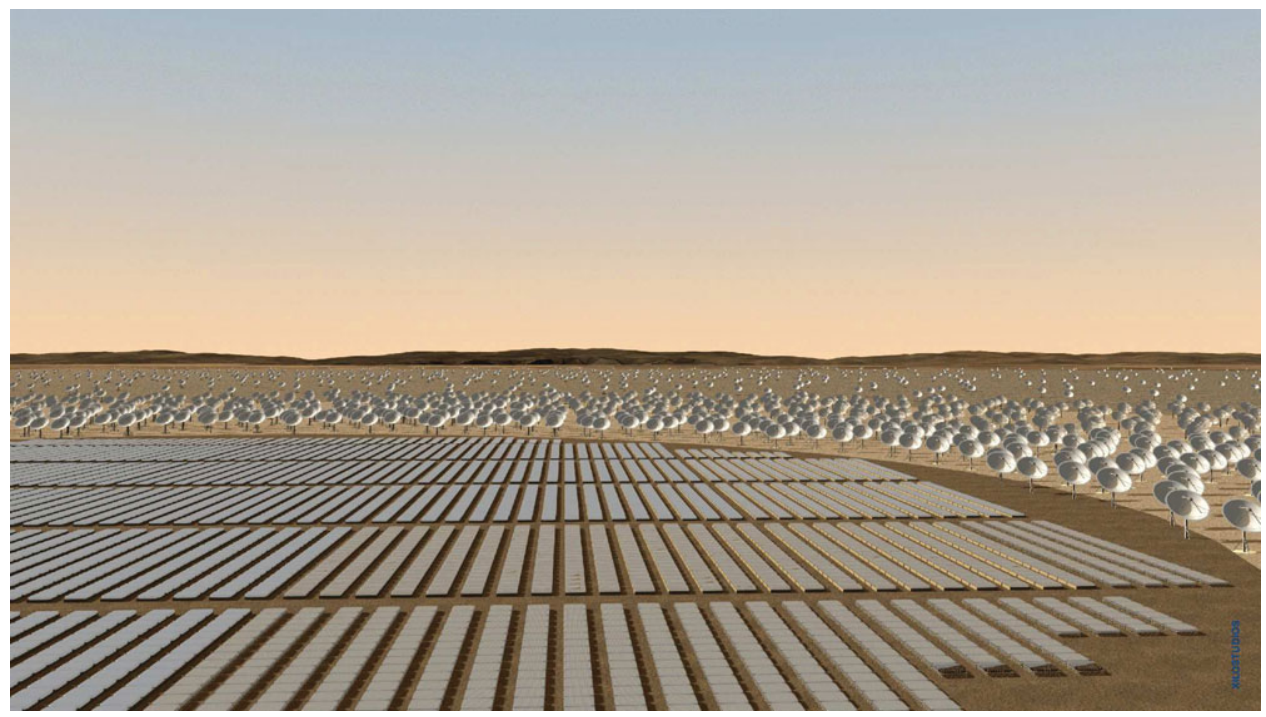

Figure 1. An artist's impression of the SKA, showing the central core of steerable dishes and passive aperture tiles. Image created by XILOSTUDIOS for the SKA Project Development Office.

\section{SKA Polarisation Pathfinders}

Although the full SKA is still some years away, a large number of pathfinder facilities are either under construction or are already beginning to take data. Many of these will be carrying out exciting new experiments on polarimetry, Faraday rotation and magnetic fields. New telescopes with such capabilities include:

- The Galactic Arecibo L-Band Feed Array Continuum Transit Survey (GALFACTS), $\dagger$ a 1.4-GHz survey that began in late-2008, and which will map the entire polarised sky visible to Arecibo;

- The Low Frequency Array (LOFAR), $\ddagger$ currently under construction in the Netherlands and Germany, which will study polarisation over the whole northern sky at very low frequencies $(\nu=30-80,110-240 \mathrm{MHz})$ (Beck 2009);

- The Allen Telescope Array (ATA) in northern California, a newly operational facility that has a wide field of view $\left(5 \mathrm{deg}^{2}\right.$ at $\left.1.4 \mathrm{GHz}\right)$ and can carry out very large continuum surveys;

- The Square Kilometre Array Molonglo Prototype (SKAMP), || a refurbishment of the Molonglo Observatory Synthesis Telescope in south-eastern Australia, which will provide $18000 \mathrm{~m}^{2}$ of collecting area for studying diffuse polarisation at an observing frequency of $\sim 1 \mathrm{GHz}$ over wide fields;

- The Murchison Widefield Array (MWA), $\dagger \dagger$ an interferometer being built in Western Australia, which will study polarised emission over wide fields in the frequency range 80-300 MHz;

- The Expanded Very Large Array (EVLA), 括, a substantial upgrade to the VLA in

$\dagger$ http://www.ucalgary.ca/ras/GALFACTS

$\ddagger$ http://www.lofar.org

๑ http://ral.berkeley.edu/ata

\| http://www.physics.usyd.edu.au/sifa/Main/SKAMP

$\dagger \dagger$ http://www.haystack.mit.edu/ast/arrays/MWA

$\ddagger \ddagger$ http://www.aoc.nrao.edu/evla 
New Mexico, providing greatly improved continuum sensitivity, frequency coverage and correlator capability;

- The Karoo Array Telescope (MeerKAT), $\dagger$ an array of 80 12-metre dishes, each equipped with a wideband feed covering the frequency range $0.7-10 \mathrm{GHz}$;

- The Australian SKA Pathfinder (ASKAP) $\ddagger$, an array of 36 12-metre antennas to be built on the Western Australian SKA site. ASKAP will be a very wide-field survey instrument $\left(30 \mathrm{deg}^{2}\right.$ at $\left.1.4 \mathrm{GHz}\right)$, and will be able to study polarisation at a range of spatial scales in the frequency range $700-1800 \mathrm{MHz}$ (Johnston et al. 2007).

\section{Cosmic Magnetism with the SKA}

The SKA key science project on cosmic magnetism focuses on three themes: structure, evolution and origin of magnetic fields (Gaensler et al. 2004). The questions we hope to address for each theme can be summarised as follows:

(a) Structure: What is the strength and structure of magnetic fields in the Milky Way, in other galaxies, and in galaxy clusters?

(b) Evolution: How have magnetic fields evolved in galaxies and clusters over cosmic time?

(c) Origin: When and how was the Universe magnetised?

In the following discussion, we outline experiments with the SKA and its pathfinders that can address each of these topics.

\subsection{Structure: The Rotation Measure Grid}

Recent surveys of polarised extragalactic sources, carried out with the Australia Telescope Compact Array and the DRAO Synthesis Telescope, have yielded background Faraday rotation data at a sky density of $\sim 1 \mathrm{RM}$ per $\operatorname{deg}^{2}$. These studies have started to reveal the large-scale magnetic field geometry of the Milky Way and Magellanic Clouds, allowing us to infer parameters such as the pitch angle of the magnetic field, the presence and location of field reversals, and the overall dynamo mode (e.g., Gaensler et al. 2005; Brown et al. 2007; Mao et al. 2008).

Observations with the SKA can spectacularly improve the sky density of background RMs. An hour of integration with the full SKA will yield approximately 2000 RMs per $\mathrm{deg}^{2}$. Over the full $20 \mathrm{deg}^{2}$ field of view, we will obtain an order of magnitude more background RMs in the first hour of observations than what has been accumulated over the last 40 years!

This will allow many exciting new applications of the "rotation measure grid". For our own Milky Way, we will be able to combine these data with pulsar RMs to derive a full three-dimensional map of the Galactic magnetic field, on scales ranging from the overall geometry of the field in the disk and halo, down to the properties of magnetised turbulence on sub-parsec scales. Deep observations of nearby galaxies will yield $>10^{5}$ RMs per target, as shown in Figure 2 - these data will allow a full reconstruction of the large-scale magnetic field in these systems. An all-sky survey of polarised continuum emission with the SKA will provide $\sim 10$ background RMs for each of the $\sim 5000$ nearest galaxies, for each of which we will be able to fit for the overall geometry and structure of the magnetic field (see Stepanov et al. 2008, for a full discussion). These studies will deliver a definitive census on the magnetic field properties of typical galaxies, and will allow us to explore how field properties (e.g., field strength, pitch angle, number

$\dagger$ http://www.ska.ac.za/meerkat

$\ddagger$ http://www.atnf.csiro.au/projects/askap 


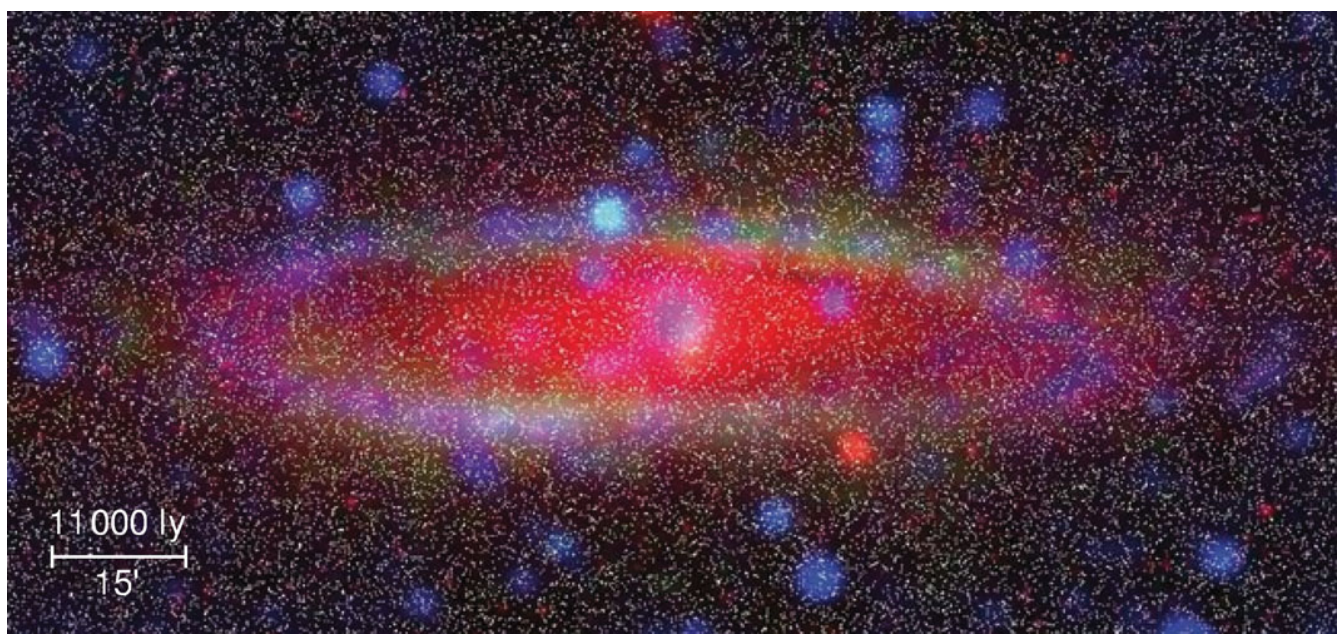

Figure 2. A simulation of the rotation measure grid behind the nearby galaxy M31, as seen with the SKA in a 30-hour observation. The colours are overlays from three observations: red indicates optical emission, blue corresponds to total intensity radio emission at $5 \mathrm{GHz}$, and green indicates polarised radio emission at $5 \mathrm{GHz}$. The white dots show the locations of 50000 simulated polarised background sources for which the SKA could extract RMs. Optical image: Digitized Sky Survey; radio images: courtesy of Rainer Beck.

of reversals) depend on parameters such as galaxy type, presence/absence of a bar, or degree of interaction.

In the lead-up to the SKA, pathfinder instruments also have an important role to play. In particular, the new wide-field sensitive surveys that will be carried out by ASKAP and the EVLA will allow us to derive catalogues of polarised extragalactic source counts down to fluxes of a microjansky or lower. The polarisation properties of these sources will allow us to separate starburst populations from low-luminosity AGNs (Taylor et al. 2007), and to characterise the statistical properties of magnetic field geometries in unresolved spiral galaxies (Arshakian et al. 2009; Stil et al. 2009).

\subsection{Evolution: Rotation Measure vs. Redshift}

For Faraday rotation at high redshifts, RMs in the observer frame will be reduced in magnitude by a factor $(1+z)^{2}$ compared to the RM in the region where rotation occurs. Thus if extragalactic RMs are dominated by Faraday rotation intrinsic to the emitting source, and if all sources have similar intrinsic RMs, then we expect that an ensemble of sources for which we have measured both RM and $z$ will demonstrate a dependence $|\mathrm{RM}| \propto(1+z)^{-2}$. However, a sample of 268 high-latitude quasars in the redshift range $0<$ $z<3.7$ show a slight increase in RM as a function of $z$ (Kronberg et al. 2008). This result, along with other new measurements of Faraday rotation (Bernet et al. 2008) and Zeeman splitting (Wolfe et al. 2008) against sources at $z \sim 0.5-2$, provides strong evidence that the observed RMs have substantial contributions from intervening absorbing galaxies, and that throughout the last $10 \mathrm{Gyr}$ of cosmic time, typical field strengths in these absorbers have been at the level of a few microgauss or more. This is at odds with the expectation from mean-field dynamo theory that the galactic magnetic fields observed today have steadily grown over time, and favours models in which the field undergoes relatively rapid amplification.

Current instrumental capabilities introduce three limitations on these intriguing new results. First, since the observed RM from an extragalactic source is usually dominated 
by the contribution from the Milky Way's interstellar medium (ISM), any correlation between RM and $z$ must use not the total observed RM, but the residual rotation measure (RRM), i.e., the RM after a contribution from the Galactic foreground has been estimated and subtracted. Currently, the relatively spare sampling of RMs over the sky limits the accuracy with which we can calculate RRMs (Dineen \& Coles 2005; Short et al. 2007). The greatly improved RM grid data that will come from pathfinder surveys with ASKAP and the ATA will provide a dramatic improvement in our ability to model and remove the Galactic foreground. The corresponding RRMs will have much smaller uncertainties.

Second, we simply lack the sample sizes needed to build up sufficient statistics of RM vs. $z$. An all-sky polarisation survey with the SKA down to sub- $\mu$ Jy sensitivities will yield $\approx 10^{8}$ background RMs. Provided that a few percent of these sources also have spectroscopic or photometric redshifts, we will be able to invert the distribution of (RM, Dec., RM, $z$ ) to derive the magnetic power spectrum of the IGM as a function of time, out to $z \sim 3$ (Kolatt 1998; Blasi et al. 1999).

Finally, if indeed the evolution of magnetic field strengths in galaxies is relatively slow over the last $10 \mathrm{Gyr}$, then we need RM data on very distant sources. Such measurements can allow us to push back to early enough times to reveal how magnetic fields in galaxies were created and amplified. Deep SKA observations of quasars or gamma-ray burst afterglows at $z>6$ can provide information on Faraday rotation at these early epochs.

\subsection{Origin: The Cosmic Web}

Deep observations of galaxy clusters at low frequencies have begun to reveal filaments and diffuse synchrotron emission seen on the periphery of, or even between, clusters (Kronberg et al. 2007; Pizzo et al. 2008). While the detections so far have been at relatively low signal-to-noise, such studies have enormous potential, because they serve as direct probes of relativistic particles and magnetic fields in the pristine intergalactic medium (IGM) (Hoeft \& Brüggen 2007; Ryu et al. 2008). Furthermore, Xu et al. (2006) have suggested that the magnetic field of the IGM might be measurable through the Faraday rotation of the background RM grid, as discussed in $§ 3.1$ above.

Observations of total intensity, polarisation and Faraday rotation with the MWA, LOFAR and SKA can extend such studies far beyond their current preliminary levels, and will provide superb insights into the magnetised large-scale structure of the Universe (Keshet et al. 2004a,b; Battaglia et al. 2009). In particular, the structure of the magnetised IGM may serve as a potential discriminant between primordial models vs. outflows as the origin of the IGM magnetic field (Donnert et al. 2009).

\subsection{Multi-wavelength Synergies}

All the above experiments will have important overlap with new experiments being carried out in other wavebands and using other techniques. At much higher radio frequencies than will be observed by the SKA, the Planck satellite will make a superb Faraday-free map of Galactic synchrotron emission and its polarisation (Enßlin et al. 2006). At optical and infrared wavelengths, forthcoming wide-field survey facilities such as SkyMapper, PanSTARRS and the LSST will provide complementary information on large-scale structure and on photometric redshifts. And finally, deflection of the trajectories of ultrahigh-energy cosmic rays measured by Auger will help map the strength and structure of magnetic fields in the local IGM. 


\section{Conclusions}

There have been many new discoveries and ideas relating to cosmic magnetism in the last few years, Using these new results as a starting point, the SKA and its pathfinders promise a suite of unique experiments aimed at revealing the structure, evolution and origin of magnetic fields in the Universe. Specifically, the dense RM grid that the SKA will provide will probe the structure of magnetic fields in the Milky Way, nearby galaxies and clusters; studies of RM vs. redshift will allow us to measure the evolution of magnetic fields in galaxies and in the IGM over cosmic time; and radio imaging of the relativistic cosmic web provides a direct view of intergalactic magnetic fields, providing information on the origin of magnetism in the Universe.

The richness of the polarised sky is already beginning to be revealed by pathfinder experiments such as GALFACTS and the ATA, with even more powerful facilities such as the EVLA and ASKAP now under construction. These activities will culminate in the next decade with the arrival of the SKA, and a consequent exploration of the full magnetic Universe. This endeavour will address major unanswered issues in fundamental physics and astrophysics, and will almost certainly yield new and unanticipated results.

\section{Acknowledgements}

I thank the organizers for financial support, and my many colleagues with whom I have collaborated on polarimetry and magnetic fields. I acknowledge the support of a Federation Fellowship from the Australian Research Council through grant FF0561298.

\section{References}

Arshakian, T. G., Beck, R., Krause, M., \& Sokoloff, D. 2009, AE\&A, in press (arXiv:0810.3114)

Battaglia, N., Pfrommer, C., Sievers, J. L., Bond, J. R., \& Ensslin, T. A. 2009, MNRAS, in press (arXiv:0806.3272)

Beck, R. 2009, in Magnetic Fields in the Universe II, in press (arXiv:0804.4594)

Bernet, M. L., Miniati, F., Lilly, S. J., Kronberg, P. P., \& Dessauges-Zavadsky, M. 2008, Nature 454,302

Blasi, P., Burles, S., \& Olinto, A. V. 1999, ApJ 514, L79

Brown, J. C., Haverkorn, M., Gaensler, B. M., Taylor, A. R., Bizunok, N. S., McClure-Griffiths, N. M., Dickey, J. M., \& Green, A. J. 2007, ApJ 663, 258

Carilli, C. \& Rawlings, S. 2004, New Astron. Rev. 48, 979

Dineen, P. \& Coles, P. 2005, MNRAS, 362, 403

Donnert, J., Dolag, K., Lesch, H., \& Müller, E. 2009, MNRAS, in press (arXiv:0808.0919)

Enßlin, T. A., Waelkens, A., Vogt, C., \& Schekochihin, A. A. 2006, AN 327, 626

Gaensler, B. M. 2004, Key Science Projects for the SKA (SKA Memo Series, No. 44)

Gaensler, B. M., Beck, R., \& Feretti, L. 2004, New Astron. Rev. 48, 1003

Gaensler, B. M., Haverkorn, M., Staveley-Smith, L., Dickey, J. M., McClure-Griffiths, N. M., Dickel, J. R., \& Wolleben, M. 2005, Science 307, 1610

Hoeft, M. \& Brüggen, M. 2007, MNRAS 375, 77

Johnston, S., Bailes, M., Bartel, N., Baugh, C., Bietenholz, M., Blake, C., Braun, R., Brown, J., Chatterjee, S., Darling, J., Deller, A., Dodson, R., Edwards, P. G., Ekers, R., Ellingsen, S., Feain, I., Gaensler, B. M., Haverkorn, M., Hobbs, G., Hopkins, A., Jackson, C., James, C., Joncas, G., Kaspi, V., Kilborn, V., Koribalski, B., Kothes, R., Landecker, T. L., Lenc, E., Lovell, J., Macquart, J.-P., Manchester, R., Matthews, D., McClure-Griffiths, N. M., Norris, R., Pen, U.-L., Phillips, C., Power, C., Protheroe, R., Sadler, E., Schmidt, B., Stairs, I., Staveley-Smith, L., Stil, J., Taylor, R., Tingay, S., Tzioumis, A., Walker, M., Wall, J., \& Wolleben, M. 2007, PASA 24, 174

Keshet, U., Waxman, E., \& Loeb, A. 2004a, ApJ 617, 281

Keshet, U., Waxman, E., \& Loeb, A. 2004b, New Astr. 48, 1119

Kolatt, T. 1998, ApJ 495, 564 
Kronberg, P. P., Bernet, M. L., Miniati, F., Lilly, S. J., Short, M. B., \& Higdon, D. M. 2008, ApJ 676,70

Kronberg, P. P., Kothes, R., Salter, C. J., \& Perillat, P. 2007, ApJ 659, 267

Mao, S. A., Gaensler, B. M., Stanimirović, S., Haverkorn, M., McClure-Griffiths, N. M., StaveleySmith, L., \& Dickey, J. M. 2008, ApJ 688, 1029

Pizzo, R. F., de Bruyn. A. G., Feretti, L., \& Govoni, F. 2008, A $6 A$ 481, L91

Ryu, D., Kang, H., Cho, J., \& Das, S. 2008, Science 320, 909

Schilizzi, R., Dwedney, P. E. F., \& Lazio, T. J. W. 2008, Proc. SPIE 7012, 52

Short, M. B., Higdon, D. M., \& Kronberg, P. P. 2007, Bayesian Analysis, 2, 665

Stepanov, R., Arshakian, T. G., Beck, R., Frick, P., \& Krause, M. 2008, A\& $A$ 480, 45

Stil, J. M., Krause, M., Beck, R., \& Taylor, A. R. 2009, ApJ, in press (arXiv:0810.2303)

Taylor, A. R., Stil, J. M., Grant, J. K., Landecker, T. L., Kothes, R., Reid, R., Gray, A. D., Scott, D., Martin, P. G., Boothroyd, A., Joncas, G., Lockman, F. J., English, J., Sajina, A., \& Bond, J. R. 2007, ApJ 666, 201

Wolfe, A. M., Jorgenson, R. A., Robishaw, T., Heiles, C., \& Prochaska, J. X. 2008, Nature 455, 638

Xu, Y., Kronberg, P. P., Habib, S., \& Dufton, Q. W. 2006, ApJ 637, 19

\section{Discussion}

KronberG: The "Extended Very Large Array (EVLA) II", whose funding request was rejected, would serve as an obvious nucleus of a future "northern SKA" operating at the higher frequencies.

DE Gouveia DAL PINO: After this excellent talk on SKA and the coming projects for cosmic magnetism search, I'd like to make a comment that there is presently a project for building a millimeter VLBI apparatus in the southern hemisphere. It is an ArgentinianBrazilian collaboration for installing two antennas in desert areas in Argentina at distances of $\sim 100 \mathrm{~km}$ from ALMA, allowing VLBI with ALMA at mm frequencies.

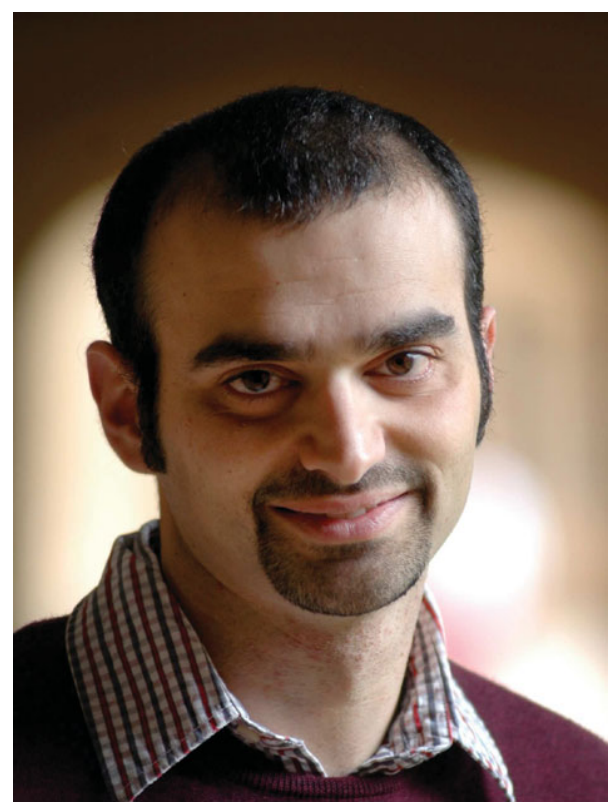

Bryan Gaensler 


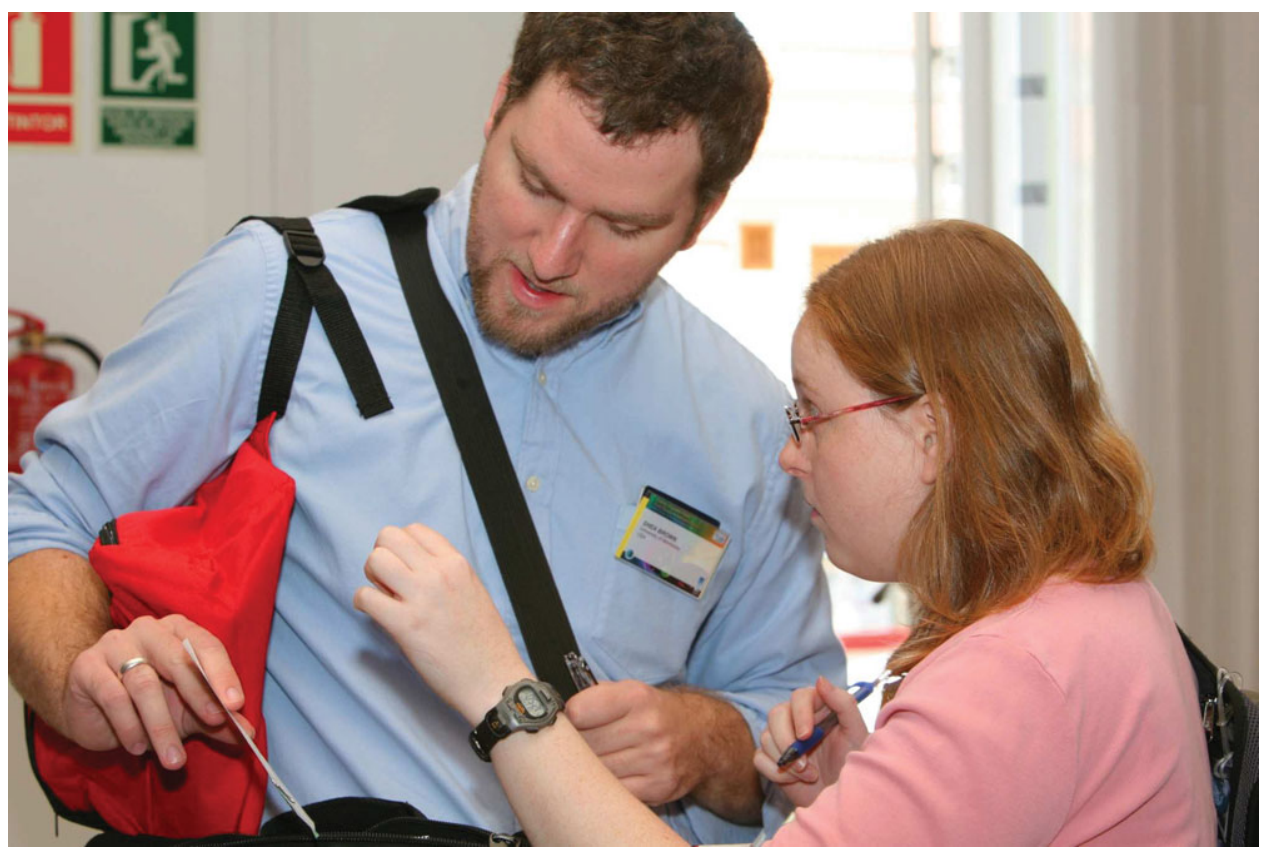

Shea Brown (left), and Amanda Kepley

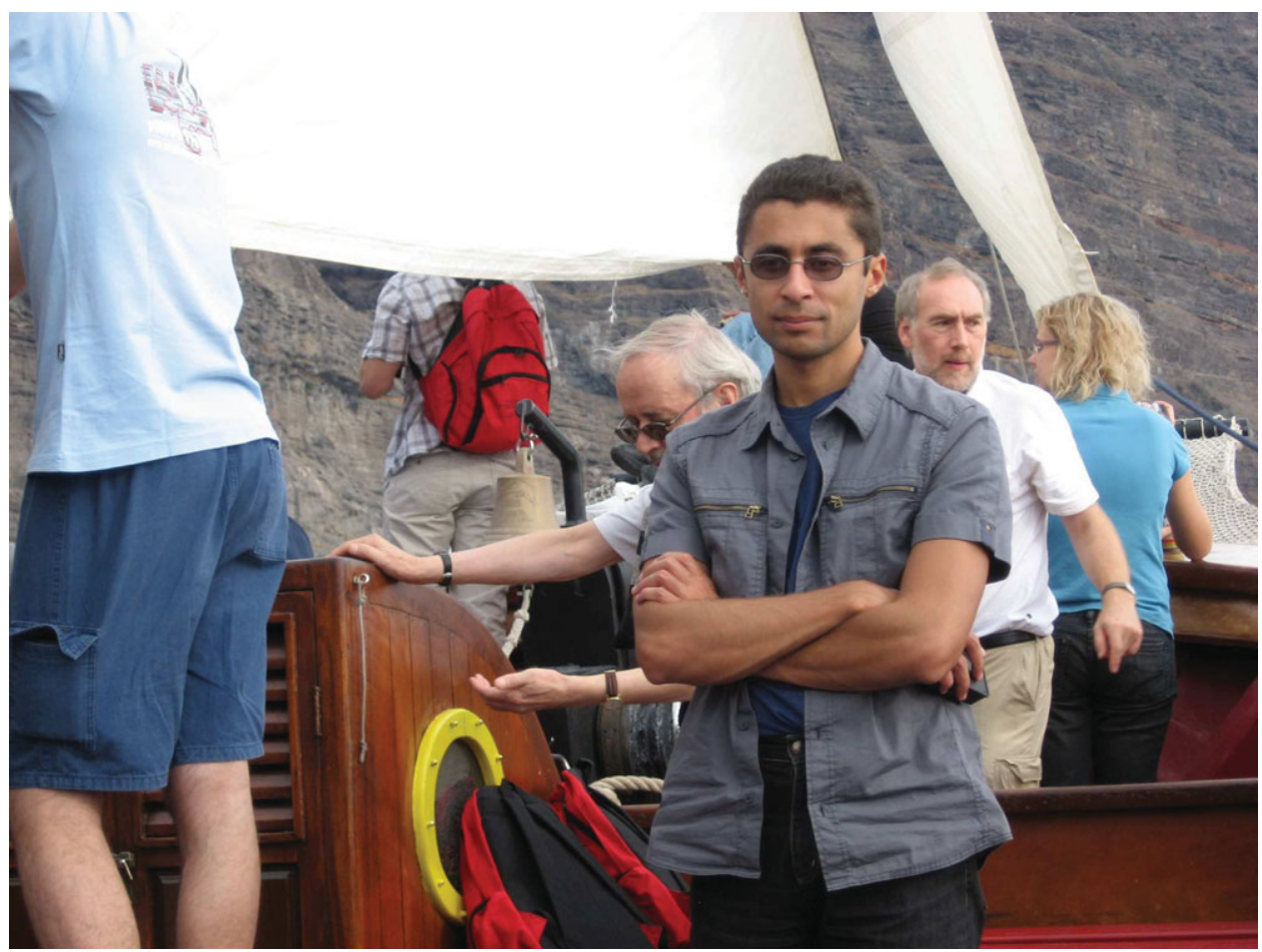

Oleg Kochukhov (facing the photographer) 